\title{
A mobile architecture with dynamic message filtering for emergency response environments
}

\author{
Bill Karakostas
}

Correspondence: billk@soi.city.ac.uk School of informatics, City

University, London, UK

\begin{abstract}
This paper demonstrates that contemporary smartphones act as effective message filtering systems in high traffic environments such as emergency response organisations, without relying on central servers. We have prototyped a mobile messaging application for Android smartphones in the Erlang language. We implemented filtering rules based on message origin, importance, and temporal validity, and tested the filtering capabilities of a smartphone in a realistic setup, that simulates traffic of tens of thousands of messages per minute, as in a large scale emergency response operation. The conclusion is that careful coding of the messaging application so that it operates in constant memory space and judicious use of the available display area can provide an effective portable message filtering for real-time, high-volume traffic, and the potential to reduce information overload for the emergency responder.
\end{abstract}

Keywords: Emergency response, Dynamic message filtering, Erlang, Mobile phones, Smartphones, Messaging simulation

\section{Introduction}

It is argued that the chief challenge for the emergency response organisation is not the scarcity of information, but its excess: too much information can strain the capacity of both the infrastructure and of the decision making functions of the organisation [1]. Experience in emergency management has shown that accurate and timely information is as crucial as is rapid and coherent coordination among the responding organizations [2].

In heterogeneous and ad-hoc emergency teams, it is difficult for the Control Room to have an accurate and up to date picture of the composition and activities of the response teams. Thus, much of the communication will be broadcasted rather than targeted to individuals, resulting in high volumes of traffic that need to be filtered by the emergency responders. We argue that mobile phone technology can assist in such a task.

The research question addressed by this paper therefore, is whether instant messaging technologies on mobile devices can be an effective means to filter in real-time through the large number of message exchanges amongst the members of an emergency response organisation. In this context, 'effectiveness' means that the messages are received and read in a timely manner, and most importantly, understood by the emergency responder and acted upon.

The paper is structured as follows. The next section surveys technologies used for emergency response, and the challenges that message overloading presents in such a 
context. It also discusses filtering as a mechanism to keep the size of message queues manageable. Section 3 discusses message-based communications in emergency response organisations, and then it proposes a message filtering approach to avoid information overload of emergency responders, implemented on a mobile device. The next section describes the results of simulating the prototype system in conditions that simulate messages in an emergency response environment. Finally, in Section 5 we propose improvements to the described approach.

\section{Technologies to support emergency response}

Various types of information and communication technologies have been trialled, aiming primarily to support emergency response planning [3]. Recent approaches [4] have started to address coordination during the emergency response execution phase through situation awareness, i.e., the ability to identify, process, and comprehend the critical elements of information about what is happening to the emergency response team with regards to the mission. Such approaches realise that most emergency responders are mobile, hence support technologies must explicitly address the mobility aspect, through location and context-aware messaging. However, use of mobile messaging in emergency response might not be without any pitfalls, as evidenced by symptoms of information overload, fatigue and anxiety, caused by the rapid growth of messaging in recent years, both on the Internet and through telephony [5]. As a result, managing and controlling the large volumes of electronic message flows is now a major commercial and research endeavour [6]. Several electronic message filtering prototypes have been implemented, such as in [7], which prioritizes voice and text messages using personal information found in the user's personal information management applications such as his/her e-calendar.

\section{Communication patterns and filtering in emergency response}

Typically, emergency response organisations are hierarchical as in the diagram of Figure 1. Teams are also usually structured as shallow hierarchies with a team leader and a group

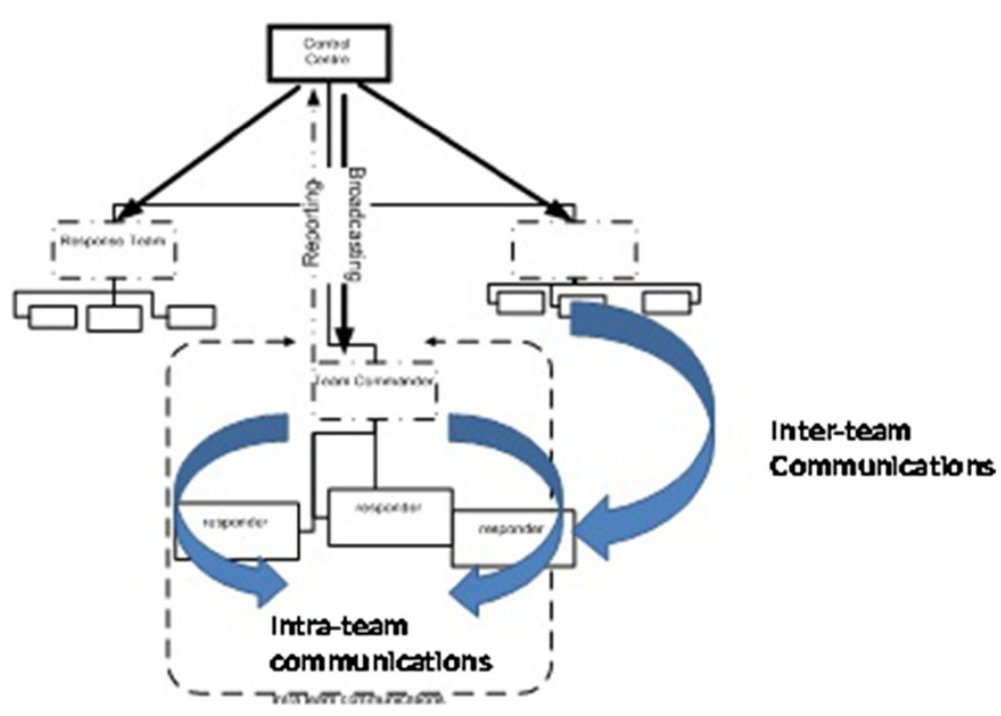

Figure 1 Communication flows in an emergency response organisation. 
of emergency responders. In emergency response, a Command and Control $(C \& C)$ Centre coordinates the actions of the emergency teams. Flow of communication is mostly top down, i.e., from the $C \& C$ to the teams, as well as amongst team members, rather than inter-team, as teams tend to communicate with each other through the $C \& C$. The $C \& C$ centre cannot always be aware of the membership composition of response teams, which also, can be dynamic, i.e., dependent on the type and status of the incident. Hence, many messages will be broadcast and so received by all members of all teams. Publish-subscribe messaging architectures can be employed to reduce the need for broadcasting. However, current implementations of publish/subscribe systems do not perform well in highly mobile and unreliable wireless settings [8]. Also, filtering technology employed in the typical publish/subscribe model has drawbacks such as unused connections and wasted queue space [9]. To avoid information overload, it is important therefore, that the emergency responder is presented with only a small subset of the message flow at any time, containing the most relevant messages, as emergency responders will have only a limited span of attention to their mobile devices.

Moreover, due to task and environmental conditions, responders might have limited spare cognitive capacity to deal with large numbers of messages. They might, for example, ignore relevant messages if they do not match their current mental model of the situation. This can happen in particular to novice responders, while experienced responders have a more developed cognitive capacity to shift through irrelevant information. Important and relevant messages must be therefore made prominent through filtering mechanisms, in order to capture the user's attention. Filtering can be implemented by assigning weights to each message and then by sorting the message list by the message weight. Weights can be based on the geographical proximity of the sender to the receiver, their organisation relationship (e.g. commander to team-member), the frequency of communications between sender and receiver, and other such criteria. Our approach employs a filtering mechanism that processes messages in real-time, prioritising the most important over a sliding time window, which means that, unlike in a typical messaging system, message importance has a temporal dimension, decreasing as the message ages, with messages outside the sliding window getting discarded. This filtering mechanism discriminates in favour of messages that are both recent and important, and keeps them in a prominent visual position, i.e., near the top of the message queue as long as they remain within the sliding time window.

\section{Design of a mobile message filtering application}

Our prototype message filtering system is implemented as a single Erlang application, running on an Android smartphone, and comprising a number of subsystems (modules) for message receiving, queuing, filtering and displaying. All messaging is carried between the mobile device, and a PC that emulates the Command and Control Centre, using TCP/IP sockets.

The main issue in the design of the message display interface is to establish how many messages could and should be visible to the emergency responder who is going to have a limited attention span. This number is of course limited by the physical dimensions and resolution of the mobile device screen. The design trade-off was to fit all messages over a weight threshold on the screen at the same time so as to avoid time consuming operations like scrolling, while keeping the number of visible messages to below a maximum size, in order not to overload the responder. We have been experimenting with Twitter style 
messages that have a size limit of 140 characters. On the character based screen of $80 \times 25$ characters used by our prototype, this makes it possible to display approximately 10 messages, after allowing for header information and white spaces.

\section{Experimental simulation}

Messages are represented as Erlang tuple data structures that consist of message identifier, a sender identifier, a message body and a timestamp. We implemented two message filtering rules that apply the principle of combining static with temporal filtering, as discussed in Section 3. Both rules were coded so that they execute in constant memory space. This makes possible to handle high volumes of message traffic without exceeding the phone's available memory. All received messages are stored in an ordered list that is reordered each time a new message is received, using a modified version of the Quicksort algorithm. The list is pruned periodically by applying the sliding time window principle, i.e., removing broadcast messages over 2 minutes old and team messages over 5 minutes old.

Another design objective was to ensure that the messaging system can cope with high volumes of constant message traffic, but also with occasional bursts, without dropping or failing to filter messages. We model the background communication message flow as a random uniform distribution where the number of inter-team messages transmitted by the network grows linear with the size of the emergency response organisation. Thus, for an organisation of size $\mathrm{n}$ we simulate a message traffic of an average of $n / 2$ messages per minute. For an organisation of 1000 members, this would amount to an average of 500 messages per minute, or approximately 8.3 messages per second. In addition to broadcast messages, we simulate intra-team messages. We assume that the arrival of such messages follows a Poisson rather than a uniform distribution. We have experimented with an emergency response team size of 10, and with event frequencies $\lambda$, ranging from 1 event per minute to 1 event per 10 minutes. When an event occurs, we simulate the intra-team communications with a random burst of between 0 and $n^{2}$ messages per $\lambda$, which for a team size of 10 and $\lambda=10$, equates to an average rate of 50 messages per 10 minutes. We carried out repeated simulations by varying the values of parameters discussed in the previous section. Each simulation run takes place in real-time and lasts for 120 minutes. For an organisation size of 1000, an average of 60,000 messages are sent to the mobile during each simulation run. Messages are auto-generated by the simulation environment, by selecting at random, a message sender, a message body from a list of preset choices, and by time-stamping the message. The refresh rate of the message list was set to between 0.2 and 1 updates per second. Although if it is unlikely that a responder is going to check her phone every few seconds for updates, a high refresh rate means that when she decides to do so she will obtain the most up to date list of the messages.

A message list update begins with a header that contains the time of the update and the total number of new messages, followed by the filtered list of messages (if any). Each message consists of the time it was received (thus showing how recent the message is), followed by the team name of the sender, and then by the message body. Figure 2 shows a message sent by a member of another team ('otherbuddy6') informing that 'the staircase in Zone 2 has collapsed'. Due to the filtering methods applied, both the identity of the sender as well as the subject of the message will be relevant to the responder's current mental state, thus helping her to maintain situation awareness. 


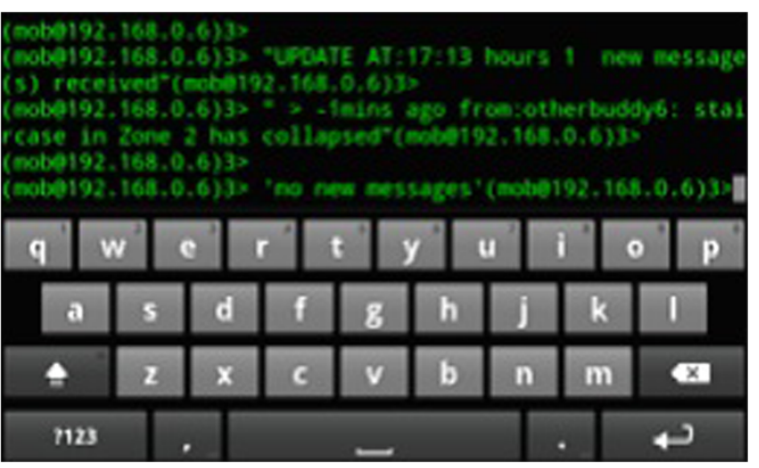

Figure 2 Message list.

Throughout the entire simulation, no message loss or incorrect filtering of received messages was observed. Thus, for the implemented set of filtering rules and sustained message rate (an average of 8 messages per second), both reflecting realistic emergency response conditions, the mobile phone seems capable of coping with both message reception and filtering.

\section{Discussion and conclusions}

Collaboration technologies for emergency response have not received much dedicated attention from research in CSCW, mobile, ubiquitous computing, and related areas; nevertheless some of the outputs of such research could be relevant to emergency response support. For example, location-based notifications that alert the users in a target area to information of interest to them could easily be added to our approach as additional types of filtering rules that add location to the properties of a received message. Filtering on the other hand, has been an active topic for research in mobile based communications. In [10] the use of a 'middleman' between message sender and receiver is proposed to filter or delay all but the most essential data when the communication link to the mobile device is slow. Yet, other approaches suggest that such filtering should be done collaboratively, i.e., shared between the participating mobile devices [11]. As this paper demonstrates, it is also feasible to do message filtering, in real-time, on individual mobile devices.

On the device we used for the experimentations, we observed a 50\% CPU utilisation during the simulation. Careful tuning of applications and services that run on the phone is required therefore, in order not to over-tax the phone's resources. More importantly perhaps, the battery utilisation of the filtering application during the simulation was observed to be in excess of $50 \%$. Hence, there is a need to formulate a filtering mechanism that optimizes the computation and space constraints [12] and reduces battery consumption, to make mobile phones suitability for long-running operations.

Future research could improve the current approach in several ways, for example, by adding multimodal interfaces such as sound and haptics, as in the multimodal information assistant system for crisis management, proposed in [13]. Such mechanisms could be employed to highlight the importance of messages, rather than relying solely on their ranking on the message list. Also, a filtering approach that uses ontologies about events, locations and emergency equipments, to perform semantic filtering of messages, 
could be utilised [12]. Finally, the filtering mechanism could be made more adaptable using either supervised or unsupervised machine learning techniques.

Received: 19 June 2012 Accepted: 7 February 2013

Published: 12 February 2013

\section{References}

1. Manoj BS, Baker AH (2007) Communication challenges in emergency response. CACM 50:3

2. Van de Walle B, Turoff M (2007) Emergency response information systems: emerging trends and technologies. CACM 50:3

3. Fiedrich F, Burghardt P (2007) Agent-based systems for disaster management. CACM 50:3

4. Karakostas B (2009) Context aware mobile coordination system for emergency response. Journal of Emergency Management 7:4

5. Bawden D, Robinson $L$ (2009) The dark side of information: overload, anxiety and other paradoxes and pathologies. J Inf Sci 35:180

6. Catone J (2009) Twitter better: 20 ways to filter your tweets July 3, 2009., Available from http://mashable.com/2009/ 07/03/twitter-filter/

7. Marx M, Schmandt C (1996) CLUES: dynamic personalized message filtering, Proc. CSCW'96 Proceedings of the 1996 ACM conference on computer supported cooperative work

8. Faroog U, Shikharesh M, Parsons EW (2004) Engineering mobile wireless publish/subscribe systems for high performance, Proceedings of the IEEE computer Society's 12th annual international symposium on modeling, analysis, and simulation of computer and telecommunications systems (MASCOTS'04)., Washington, DC, USA, pp 295-305

9. Kim SW, Cho KB, Seungwok Han H, Yong Y (2007) Efficient and dynamic location-based event service for mobile computing environments, Fifth international conference on computational science and applications, (ICCSA 2007)., Kuala Lumpur, Malaysia, August 26-August 29

10. Zenel B, Duchamp D (1995) Intelligent communication filtering for limited bandwidth environments, Proceedings of the fifth workshop on Hot topics in operating systems (HotOS-V)

11. Gratz P, Andronache A, Rothkugel S (2008) Ad Hoc collaborative filtering for mobile networks, IEEE international conference on sensor networks., Ubiquitous, and Trustworthy Computing SUTC '08 11-13 June

12. Lia LH, Leea F-M, Yu-Chien C, Tsung-Jen P (2010) Using ontology for personalized mobile message computation, 24th IEEE international conference on advanced information networking and applications(AINA) 20-23 April

13. Cao Y, Nijholt A (2008) Modality planning for preventing tunnel vision in crisis management, Symposium on multimodal output generation (MOG 2008) at the AISB 2008 convention. Communication, Interaction and Social Intelligence, Aberdeen, UK, 3-4 April

doi:10.1186/2192-1121-2-2

Cite this article as: Karakostas: A mobile architecture with dynamic message filtering for emergency response environments. Communications in Mobile Computing 2013 2:2.

Submit your manuscript to a SpringerOpen ${ }^{\circ}$ journal and benefit from:

- Convenient online submission

- Rigorous peer review

- Immediate publication on acceptance

- Open access: articles freely available online

- High visibility within the field

- Retaining the copyright to your article

Submit your next manuscript at $>$ springeropen.com 